\title{
Inductance Extraction for General Interconnect Structures
}

\author{
Chun-Ying Lai ${ }^{1}$, Shyh-Kang Jeng ${ }^{2}$, Yao-Wen Chang ${ }^{3}$, and Chia-Chun Tsai ${ }^{4}$ \\ ${ }^{1}$ Graduate Institute of Communication Engineering, National Taiwan University, Taipei 106, Taiwann \\ ${ }^{2}$ Department of Electrical Engineering \& Graduate Institute of Communication Engineering, National Taiwan University, Taipei 106, Taiwan \\ ${ }^{3}$ Department of Electrical Engineering \& Graduate Institute of Electronics Engineering, National Taiwan University, Taipei 106, Taiwan \\ ${ }^{4}$ Department of Computer Science and Information Engineering, Nan-Hua University, Chia-Yi 622, Taiwan
}

\begin{abstract}
As the operation frequency reaches gigahertz in very deep-submicron designs, the effect of on-chip inductance on circuit performance can no longer be neglected. Therefore, it is desired to extract interconnect impedance and inductance accurately. Most previous works on impedance and inductance extraction are based on rectangular discretization which has been shown effective for the classical Manhattan based IC interconnect structures. As technology advances, however, more general IC interconnect structures, such as the X-based interconnect structure, have been in production. Those general interconnect structures allow wires to be routed with non-Manhattan shapes. For the non-Manhattan interconnect structures, rectangular discretization is obviously not sufficient. In this paper, we propose to use the surface integral formulation with triangular discretization to extract impedance and inductance for the general IC interconnect structures. Comparative studies with the well-known FastImp and IE3D show that our approach is flexible and effective.
\end{abstract}

\section{Introduction}

In high-performance circuit designs, on-chip inductance has become increasingly more significant due to faster rise times, lower resistance, and lower capacitance [2]. Wider wires are frequently encountered in clock distribution networks and in higher metal layers [10]. Those wires are low resistance lines that can exhibit significant inductive effects. Furthermore, performance requirements are pushing the introduction of new materials such as copper interconnect for low resistance interconnect and new dielectrics to reduce interconnect capacitance. These technology advancements make the inductance effect increasingly important than ever. Therefore, it is desirable to extract interconnect inductance and impedance accurately for high-performance circuits.

There exist many well-known works in the literature on accurate inductance and impedance extraction, such as FastImp [16] and FastHenry [9] [15]. Most of the previous works are based on rectangular discretization-rectangular panel discretization using the surface integral formulation [5] [16] or filament discretization [9] [15] using the volume integral formulation. For example, FastImp uses the surface integral formulation with rectangular panel discretization. In contrast, FastHenry uses the volume integral formulation with filament discretization.

The works with the rectangular discretization may effectively extract the impedance of a conductor with the Manhanttan (rectilinear) structure. However, they may not be effective enough for handling non-Manhattan interconnect structures. As technology advances, however, more general IC interconnect structures, such as the X-based [6] interconnect structure, have been introduced or even already in production by UMC. Those general interconnect structures allow wires to be routed with nonManhattan shapes. For the non-Manhattan interconnect structures, classical rectangular discretization is obviously not sufficient.

In this paper, we propose to use the surface integral formulation with triangular discretization to extract impedance and inductance for the general IC interconnect structures for high-speed circuit designs. A significant advantage of triangular discretization is that it is more flexible and powerful in geometry discretization (e.g., for extracting the inductance in the non-Manhattan X-based interconnect structures) since triangles are the most fundamental polygon and any polygon can be triangulated; therefore, it does not incur any additional difficulties for extracting impedance/inductance in the non-Manhattan interconnect structures. Note that a polygon cannot always be divided into rectangles; therefore, it is obvious that classical rectangular discretization is not sufficient for modeling the general interconnect structures such as the $\mathrm{X}$ based interconnects. Further, the surface integral formulation is generally considered more accurate than the volume integral formulation at the highfrequency domain. Comparative studies with the well-known FastImp and IE3D [11] (a commercial tool for the Electro-Magnetics [EM] solver) show that our approach is flexible and effective for extracting the impedance and inductance of general IC interconnects.

The remainder of this paper is organized as follows. Section II gives the preliminaries. Section III presents the triangular discretization. Section

$0-7803-9390-2 / 06 / \$ 20.00$ C2006 IEEE
IV shows the simulation results.

\section{Preliminaries}

We first give some preliminaries for our triangular discretization. Consider a homogeneous circuit system of multiple conductors with the constant permittivity $\epsilon$, permeability $\mu$, and conductivity $\sigma$. Let $E$ be an electric field. We use the surface integral formulation proposed in [5] [16] for our triangular discretization.

Maxwell's equations for the time harmonic field are given by

$$
\begin{aligned}
\nabla \times \vec{E}=-i \omega \mu \vec{H} ; & \nabla \times \vec{H}=i \omega \epsilon \vec{E}+\vec{J} \\
\nabla \cdot(\epsilon \vec{E})=\rho ; & \nabla \cdot(\mu \vec{H})=0
\end{aligned}
$$

where $\omega$ is the angular frequency and $e^{j \omega t}$ dependence is assumed, $\rho$ is the charge density, $H$ is the magnetic filed, and $J$ is the current density. Here, $\nabla \times$ and $\nabla \cdot$ mean curl and divergence, respectively.

By Maxwell's equations, we have

$$
\nabla^{2} \vec{A}=-\mu \vec{J} ; \quad \nabla^{2} \psi=-\rho / \epsilon,
$$

where $\nabla^{2}$ is laplacian, $A$ is the magnetic vector potential, and $\psi$ is potential on a vertex. The integral equations for $\vec{A}$ and $\psi$ are as follows:

$$
\vec{A}(x)=\int_{V} G_{0}(x, y) \mu \vec{J}(y) d y ; \quad \psi(x)=\int_{S} G_{0}(x, y) \frac{\rho}{\epsilon} d y
$$

where

$$
G_{0}(x, y)=\frac{1}{4 \pi|x-y|}
$$

$V$ is the union of all conductor volumes, $S$ is the union of all conductor surfaces, and $x$ and $y$ are position vectors. For every conductor we have a vector Helmhotz equation for $\vec{E}$ as follows:

$$
\nabla^{2} \vec{E}-i \omega \mu \sigma \vec{E}=0
$$

Let $\mathrm{P}$ and $\mathrm{Q}$ be two arbitrary vectors. Integrating Green's second vector identity [3]

$$
\begin{gathered}
\int_{V}(\vec{Q} \cdot \nabla \times \nabla \times \vec{P}-\vec{P} \cdot \nabla \times \nabla \times \vec{Q}) d V \\
=\oint_{S}(\vec{P} \times \nabla \times \vec{Q}-\vec{Q} \times \nabla \times \vec{P}) \times d \vec{S}
\end{gathered}
$$

into Equation (5), we have

$$
\int_{S} G_{1}(x, y) \frac{\partial \vec{E}(y)}{\partial n_{y}} d y-\int_{S} \frac{\partial G_{1}(x, y)}{\partial n_{y}} \vec{E}(y) d y=\vec{E}(x)
$$

where $n_{y}$ is the $y$-component of the normal vector $n$,

$$
G_{1}(x, y)=\frac{e^{i K_{1}|x-y|}}{4 \pi|x-y|}, K_{1}=\sqrt{-i \omega \mu \sigma_{k}},
$$

$\vec{P}$ and $\vec{Q}$ are replaced by $E$ and $G_{1}(x, y)$, respectively.

Applying Equation (6) into another vector Helmhotz equation

$$
\nabla^{2} \vec{E}=i \omega \mu \vec{J}
$$

we get

$$
\int_{S} G_{0}(x, y) \frac{\partial \vec{E}(y)}{\partial n_{y}} d y-\int_{S} \frac{\partial G_{0}(x, y)}{\partial n_{y}} \vec{E}(y) d y+\nabla \psi(x)=0,
$$

where $n$ is the outward normal unit vector on the conductor surface. 
So far we have derived only two equations for three unknowns $\vec{E}, \frac{\partial \vec{E}}{\partial n}$, and $\psi$, and thus we need one more equation to solve the system. Two approaches can yield the required equation: One is to enforce $\nabla^{2} \psi=0$, and the other is to enforce the current conservation

$$
\nabla \cdot E=0 \text {. }
$$

For a closed loop, both approaches are equivalent, but for an open loop $\nabla^{2} \psi=0$ is no longer true, while $\nabla \cdot E=0$ still holds. Therefore, the current conservation is enforced for all cases.

We consider a surface $S_{i}$ encircled by a closed path $C$. The thickness between the top and the bottom surfaces is a small distance quantity $\delta$, and we have the current conservation equation as follows:

$$
\begin{gathered}
\int_{C} \delta E_{t} \cdot(n(x) \times l(x)) d x= \\
\int_{t_{\alpha 1}}^{t_{\alpha 2}} \int_{t_{b 1}}^{t_{b 2}}\left(E_{n(y)}\left(t_{a}, t_{b}, 0\right)-E_{n(y)}\left(t_{a}, t_{b},-\delta\right)\right) d t_{a} d t_{b}
\end{gathered}
$$

where $t_{a 1}, t_{a 2}, t_{b 1}$ and $t_{b 2}$ are the integration boundaries of $t_{a}$ and $t_{b}, n$ is the normal vector, and $l$ is the tangetial vector of the panel side. The left-hand side is contributed by $E_{n}$ from the top and the bottom surfaces, and the right-hand side of the equation is the effect of $E_{t}$, which is the tangential $E$ field

Let $\delta$ approach zero and apply Taylor's expansion to (11). We can obtain a surface integral form of the current conservation as follows:

$$
\int_{C} E_{t}(x) \cdot(n(x) \times l(x)) d x-\int_{a} \frac{\partial E_{n}(y)}{\partial n(y)} d y=0,
$$

where $E_{n}$ is the electric field in the normal direction, and $E_{t}$ is the electric field in the tangential direction.

Finally, we deal with the boundary conditions. There could be many surfaces in a conductor. We can divide the surfaces into the contact surfaces and the non-contact surfaces. A contact surface is a surface that contacts a voltage or a current source; otherwise, it is a non-contact surface. See Figure 1 for an illustration.

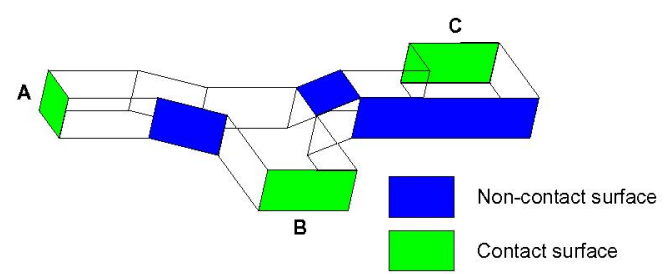

Fig. 1. Contact and non-contact surfaces.

Two boundary conditions are used for the conductor contacts. The potential at a contact is given, and it is assumed that there is no tangential current flow at the contact. As the electric field is divergence-free, $\frac{\partial E_{n}}{\partial n}$ must be zero at the contact.

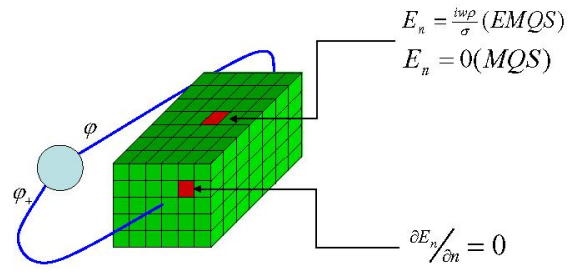

Fig. 2. Contact and non-contact surface boundary conditions.

We summarize the boundary conditions as follows (see Figure 2 for an illustration):

- Panels in the non-contact surfaces:

$E_{n}=\frac{i \omega \rho}{\sigma}$ (Electro-Magnetic-Quasi-Statics, EMQS) and

$E_{n}=0$ (Magneto-Quasi-Statics, MQS);

- Panels in the contact surfaces: apply $\frac{\partial \vec{E}_{n}}{\partial n}=0$; here, $E_{n}$ is the electrical field in the normal direction;

- Panels in the contact surfaces: $\psi$ is set to $\psi_{+}$or $\psi_{-}$.

\section{Discretization}

In order to solve the whole system, we discretize the equation. We consider that there are many surfaces in a 3-D IC interconnect. For each surface, we can discretize it into many panels and vertices.

The major difference between our work and the previous works is the way of discretization. Previous works focus on the rectangular discretization into rectangular panels or filaments [5] [16]. For the nonManhattan interconnect structures, classical rectangular discretization is obviously not sufficient. In order to effectively handle non-Manhattan interconnect structures, we propose to use triangular discretization, as illustrated in Figure 3. As mentioned earlier, the most significant advantage of using triangular discretization lies in the fact that an interconnect of any shape can be exactly divided into triangle, leading to intrinsically more accurate modeling than the classical rectangular discretization.

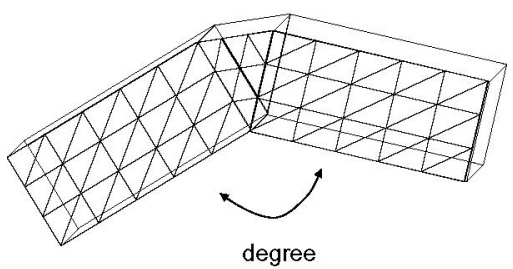

Fig. 3. Triangular discretization.

To apply the triangular discretization, we shall present a new discretization method. There are seven unknowns in a panel as follows: $E_{x}, E_{y}$, $E_{z}, \frac{\partial E_{x}}{\partial n}, \frac{\partial E_{y}}{\partial n}, \frac{\partial E_{z}}{\partial n}$, and $\rho$. Here, $x, y, z$ are the axes of the space, $n$ is the panel's normal vector, and $\rho$ is the charge density. The scalar potential $\psi$ is associated with the panel vertices.

If we use centroid collocation to discretize Equation (7), we have

$$
P_{1} \frac{\partial \vec{E}}{\partial n}-D_{1} \vec{E}=0
$$

where

$P_{1}(i, j)=\int_{\text {panel }_{j}} G_{1}\left(x_{i}, y\right) d y ; \quad D_{1}(i, j)=\int_{\text {panel }_{j}} \frac{\partial G_{1}}{\partial n}\left(x_{i}, y\right) d y$, where $x_{i}$ is the circumcenter of the $i^{\text {th }}$ panel.

Discretizing Equation (9), we can get a similar equation as above, with the only difference being with the gradient term $\nabla \psi$. For any panel, two independent tangential vectors can be found. As shown in Figure 4 , two tangential directions $t_{a}$ and $t_{b}$ are formed by connecting a node and the midpoints of the sides. The representation of the tangential $\nabla \psi$ can be obtained using finite differences. Therefore, Equation (9) in the $t_{a}$ direction can be transferred into

$$
T_{t_{a}} P_{0} \frac{\partial \vec{E}}{\partial n}-T_{t_{a}} D_{0} \vec{E}+\nabla_{t_{a}} \psi=0
$$

where

$$
P_{0}(i, j)=\int_{\text {panel }_{j}} G_{0}\left(x_{i}, y\right) d y, ; \quad D_{0}(i, j)=\int_{\text {panel }_{j}} \frac{\partial G_{0}}{\partial n}\left(x_{i}, y\right) d y,
$$

and $T_{t_{\alpha}}$ is the transfer matrix related to the local coordinate system ( $t_{a}$ or $\left.t_{b}\right)$ to the global coordinate system $(x, y$, and $z)$.

The gradient of $\psi$ along $t_{a}$ in Figure 4 can be computed by

$$
\nabla_{t_{a}} \psi=\frac{\frac{\psi_{\alpha}+\psi_{\gamma}}{2}-\psi_{\beta}}{\left|\beta M_{\alpha \gamma}\right|}
$$

where $\psi_{\alpha}, \psi_{\beta}$, and $\psi_{\gamma}$ are the voltages of nodes $\alpha, \beta$, and $\gamma$, respectively, $M_{\alpha \gamma}$ is the midpoint of nodes $\alpha$ and $\gamma$, and $\left|\beta M_{\alpha \gamma}\right|$ is the length of the line $\beta M_{\alpha \gamma}$. So we can find an $A_{t_{\alpha}}$ matrix, for which $A_{t_{\alpha}} \psi$ is equal to $\nabla_{t_{\alpha}} \psi$.

In order to generate a set of equations for the vertex potentials, we consider applying the current conservation equation (10) to Figure 5. Node $Q$ in the figure is the vertex at the intersection of the panels. First we find the circumcenter of every triangle, such as $C_{P 1}, C_{P 2}, C_{P 3}, C_{P 4}, C_{P 5}$, and $C_{P 6}$. Second, we connect these nodes to build another panel (see Figure 5), the region surrounded by dashed lines. Applying the surface integral equation (10) to this region generates the following equation:

$$
\left.\int_{P} E_{t}(x) \cdot(n(x)) \times l(x)\right) d x-\int_{S} \frac{\partial E_{n}(y)}{\partial n(y)} d y=0,
$$




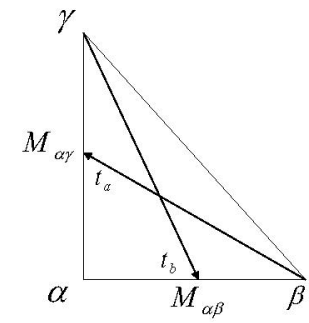

Fig. 4. Computation of the gradient of potential.

where $P$ is the perimeter of the region $S$ enclosed by the dashed lines illustrated in Figure 5. For an arbitrary point $x$ on $P, n(x)$ is the unit normal vector, and $l(x)$ is the unit vector along $P$.

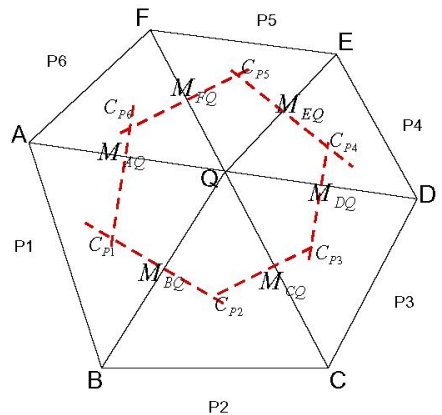

Fig. 5. Panels of current conservation.

Applying the aforementioned three boundary conditions, we can get the discretized form as follows:

$$
\begin{gathered}
N_{N C} \cdot \vec{E}_{j}=\frac{i \omega \rho_{j}}{\sigma} \quad \text { (for non-contact panel [EMQS]), } \\
N_{C} \cdot \frac{\partial \vec{E}_{j}}{\partial n}=0 \text { (for contact panel), }
\end{gathered}
$$

and

$$
F \psi=\psi_{i} \quad \text { (excitating voltage source), }
$$

where $N_{N C}$ and $N_{C}$ are matrices formed by unit normal vectors at the non-contact and contact panels, and $F$ is a matrix containing the potential information of the vertices.

We can extend Equation (13) into three formulae for the $x, y$, and $z$ directions. Nevertheless, Equation (14) can only be extended into two formulae because there are only two independent tangential vectors on the panels, $t_{a}$ and $t_{b}$. According to the above formulae, the system matrix can be derived as shown in Figure 6.

After solving the system matrix by an iterative method, the coefficients of all parameters in each panel can be found. Then we can compute the incident current accordingly by the following equations:

$$
J=\sigma E, \quad I=\int J d s=\sum_{\text {panel }_{j} \in C_{+}}-\sigma\left(n_{j+} \cdot E_{j}\right) s_{j},
$$

where $I$ is the incident current illustrated in Figure $7, J$ is the current density, $n_{j+}$ is the normal vector of panel $_{j}$ (pointing outwards), $E_{j}$ is the electric field at panel ${ }_{j}, s_{j}$ is the area of panel $_{j}$, and panel $_{j}$ belongs to one of the contact surfaces $C_{+}$.

In the boundary conditions, we make the voltage drop between two terminals $1 \mathrm{~V}$. Therefore, we can compute the impedance $Z$ by

$$
Z=\frac{1}{I}
$$

and the inductance $L_{\text {eff }}$ by

$$
L_{e f f}=\frac{i \operatorname{mage}(Z)}{\omega} .
$$

\section{Simulation Results}

In this section, we present numerical simulation results by using the aforementioned formulation to perform both magneto-quasi-static and electromagnetic analysis for several types of interconnect structures. Our comparative studies are based on public solvers, FastImp and the commercial electromagnetics (EM) tool, IE3D.

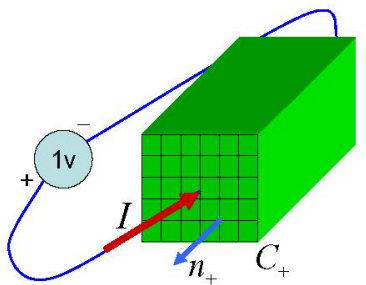

Fig. 7. Current computation.

\section{A. Straight Interconnects}

To verify the accuracy of our solver, we first performed simulations on a straight interconnect of $1 \mathrm{~mm}$ wide, $1 \mathrm{~mm}$ high, and $4 \mathrm{~mm}$ long, to which the tools FastImp, and IE3D can apply. Let $\sigma=5.8 \times 10^{7} \mathrm{mho} / \mathrm{m}$ (for copper interconnects). In the IE3D tool, the substrate's $\sigma$ could be set as a huge number so that the substrate is treated as a perfect conductor. Then we can transform $S$ parameters into $Z$ parameters. The simulated inductances are compared based on various frequencies ranging from 100 $\mathrm{MHz}$ to $10 \mathrm{GHz}$ by using FastImp with 128 panels, IE3D, and our solver.

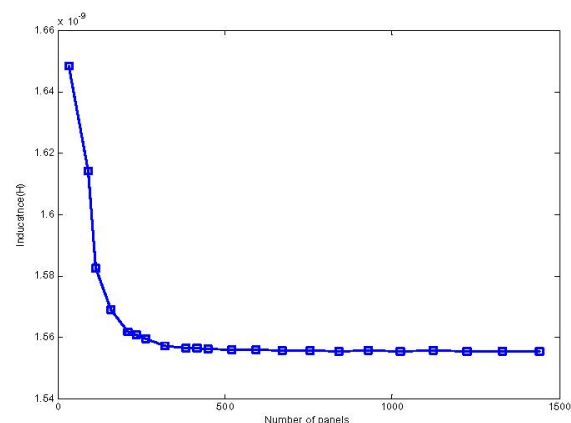

Fig. 8. The relationship between the value of inductance and the number of discretized panels

The results show that the curves of FastImp, IE3D, and our solver well match each other. Especially, the curve of our solver is almost overlapped with that of FastImp. The reason is that the above three programs, FastImp, IE3D, and ours, all use the surface integral formulation. In addition, FastImp and ours are independent of the frequency while IE3D changes gradually with frequency. The reason is that we linearize our formulation at low frequency, same as FastImp. The results reveal that our solver with triangular discretization is sufficiently accurate in the inductance and resistance extractions for straight interconnects. Figure 8 shows that the inductance (simulation results) converges when the number of discretized panels increases.

\section{B. X-Based Interconnects}

The second experiment intends to test the accuracy of our solver on the X-based interconnect structure, to which most solvers with rectangular discretization cannot apply directly. See Figure 9 for an interconnect between nodes $A$ and $B$ with a 45 -degree turn (i.e., an $\mathrm{X}$-based structure). We set the operation frequency to $3 \mathrm{GHz} . A$ and $B$ are the diagonal vertices on a $6 \mathrm{~mm} \times 6 \mathrm{~mm}$ square.

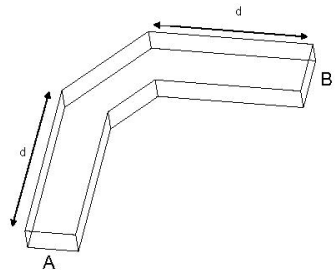

Fig. 9. An L-shaped conductor.

Figure 10 shows the simulation results for inductance by changing the value $d$ from $1 \mathrm{~mm}$ to $6 \mathrm{~mm}$. We compared the results with IE3D. As shown in the figure, the behavior of our solver is very similar to that of the IE3D EM solver. It should be noted that the IE3D does not scale to 


\begin{tabular}{|c|c|c|c|c|c|c|c|c|c|c|}
\hline$P_{1}$ & 0 & 0 & $-D_{1}$ & 0 & 0 & 0 & 0 & & & 0 \\
\hline 0 & $P_{1}$ & 0 & 0 & $-D_{1}$ & 0 & 0 & 0 & {$\left[\frac{\partial E_{x}}{\partial n_{n}}\right.$} & & 0 \\
\hline 0 & 0 & $P_{1}$ & 0 & 0 & $-D_{1}$ & 0 & 0 & $\frac{\partial E_{y}}{\partial p}$ & & 0 \\
\hline$T_{t a_{\infty}} P_{0}$ & $T_{t a_{y},} P_{0}$ & $T_{t a_{z}} P_{0}$ & $T_{t a_{x}}\left(D_{0}-I\right)$ & $T_{t a_{t}}\left(D_{0}-I\right)$ & $T_{t a_{z}}\left(D_{0}-I\right)$ & $A_{t_{\infty}}$ & 0 & $\frac{\partial m_{z}}{\partial m}$ & & 0 \\
\hline$T_{t b_{x}} P_{0}$ & $T_{t b_{y}} P_{0}$ & $T_{t b_{z}} P_{0}$ & $T_{t b_{w}}\left(D_{0}-I\right)$ & $T_{t b_{s}}\left(D_{0}-I\right)$ & $T_{t b_{z}}\left(D_{0}-I\right)$ & $A_{t_{b}}$ & 0 & ${ }_{E_{x}}^{\partial n}$ & $=$ & 0 \\
\hline 0 & 0 & 0 & $N_{N C_{x}}$ & $N_{N C_{y}}$ & $N_{N C_{z}}$ & 0 & $-\frac{i \omega}{\sigma}$ & $E_{y}$ & $=$ & 0 \\
\hline$N_{C_{x}}$ & $N_{C_{y}}$ & $N_{C_{x}}$ & 0 & 0 & 0 & 0 & $0^{\circ}$ & $E_{z}$ & & 0 \\
\hline$C_{d_{x}}$ & $C_{d_{t}}$ & $C_{d_{z}}$ & $C_{x}$ & $C_{y}$ & $C_{z}$ & 0 & 0 & $\psi$ & & 0 \\
\hline 0 & $0^{\circ}$ & 0 & 0 & 0 & 0 & $F$ & 0 & $\rho$ & & $\psi_{i}$ \\
\hline 0 & 0 & 0 & 0 & 0 & 0 & $-\epsilon A_{p}$ & $P_{0}$ & & & 0 \\
\hline
\end{tabular}

Fig. 6. The system matrix.

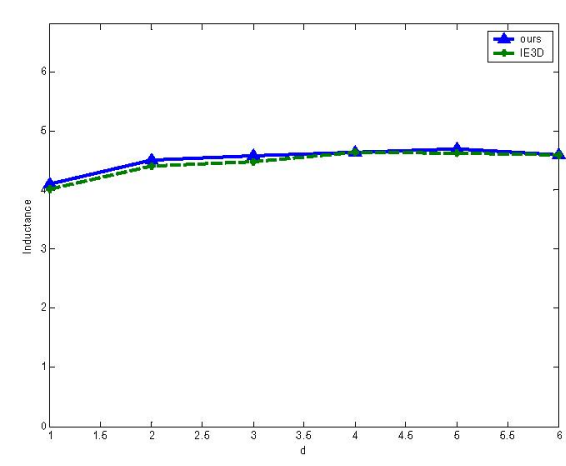

Fig. 10. The relation between the inductance and the d value shown in Figure 9.

large-scale interconnects since it is an EM solver which is intended for analyzing a layout configuration with few interconnects. Further, the user needs to manually draw every physical interconnect for IE3D simulation, which is tedious and human-labor intensive. Therefore, the IE3D software is not feasible for on-chip interconnect designs.

\section{Arbitrarily Shaped Interconnects}

The last experiment explores how to approximate the inductance for an arbitrarily shaped interconnect. As shown in Figure 11, we intend to make a connection between nodes $A$ and $B$ located along a diagonal in a square of $6 \mathrm{~mm} \times 6 \mathrm{~mm}$ with obstacles. For a non-Manhattan interconnect, we can use an appropriate point $C$ in the region $\triangle A D B$ as a corner to prevent the connection from passing through the obstacles. We intend to explore the relation between the position of $C$ and the corresponding value of inductance. Let the operation frequency be $1 \mathrm{GHz}$.

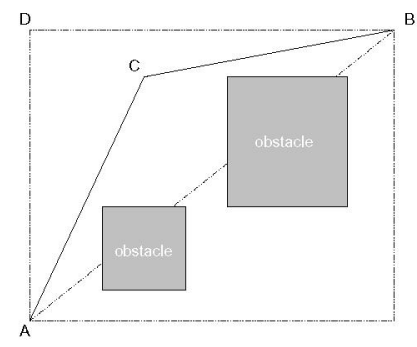

Fig. 11. An interconnect of arbitrary shape.

In Figure 12, the inductance (in $n H$ ) is plotted as a function of the ratio $\triangle A C B / \triangle A D B$. From the figure, we observe that the diagonal interconnect gives the minimum self-inductance while the right-angle $(L$ shaped) interconnect induces the maximum inductance. Though not shown here, different triangles $\triangle A C B$ 's with the same area give approximately the same inductance. Further, the value of inductance is proportional to the area of $\triangle A C B$. Therefore, the function given in Figure 12 provides a reasonable estimation for the inductance of an arbitrarily shaped interconnect.

\section{Conclusion}

In this paper, we have proposed a surface integral formulation with triangular discretization to compute EMQS and MQS inductance and resistance for general interconnect structures. The major advantage of this discretization lies in its high flexibility in extracting the inductance

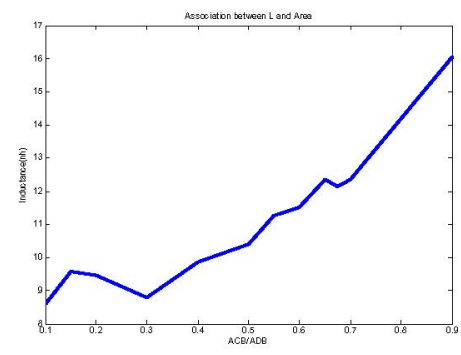

Fig. 12. The relationship between the inductance value and the ratio $\triangle A C B$

and impedance for general interconnect structures, such as the X-based interconnects. Another advantage is its high fidelity for inductance and impedance extraction in the high-frequency domain.

\section{Acknowledgments}

This work was partially supported by National Science Council of Taiwan under Grant No's. NSC 93-2215-E-002-009 and 93-2752-E-002008-PAE.

\section{References}

[1] K. Banerjee and A. Mehrotra, "Accurate Analysis of On-Chip Inductance Effects and Implications for Optimal Repeater Insertion and Technology Scaling," IEEE Symp. VLSI Circuits, pp. 195-198, June 2001.

[2] A. Deutsch, A. Kopcsay, and G. V. Surovic, "Challenges Raised by Long On-Chip Wiring for CMOS Microprocessors," Proceedings of IEEE Topical Meeting on Electrical Performance of Electronic Packaging, pp. 21-23, Oct. 1995.

[3] A. Guissard, "On the Surface Field Integral Equations," IEEE Trans. Education, vol. 46, no. 4, Nov. 2003.

[4] L. He, et al, "An Efficient Inductance Modeling for On-chip Interconnects," Proceedings of the IEEE Custom Integrated Circuits Conference, pp. 457-460, May 1999.

[5] J. Huang, B. song, J. K. White and Z. Z. Hu, "Improving the Robustness of a Surface Integral Formulation for Wideband Impedance Extraction of 3D Structures." Proc. of the IEEE/ACM ICCAD, Nov. 2001

[6] M. Igarashi, T. Mitsuhashi, A. Le, S. Kazi, Yang-Trung Lin, A. Fujimura, and S. Teig, "A diagonal-interconnect architecture and its application to RISC core design," Proc. of the IEEE ISSCC, vol. 1, pp. 210-460, 2002.

[7] Y. I. Ismail and E. G. Friedman, "Equivalent Elmore Delay for RLC Trees," IEEE Trans. Computer-Aided Design, vol. 19, no. 1, pp. 83-97, Jan. 2000.

[8] A. B. Kahng and S. Muddu, "An Analytical Delay Model for RLC Interconnects", IEEE Trans. Computer-Aided Design, vol. 16, no. 12, pp. 1507-1514, Dec. 1997.

[9] M. Kamon, M. J. Ttsuk and J. K. White,"FASTHENRY: A Multipole-accelerated 3-D Inductance extracion Program," IEEE Trans. Microwave Theory and Techniques, vol. 42 , Issue 9, pp. 1750-1758, Sept. 1994

[10] I. S. Kourtev and E. G. Friedman, Timing Optimization Through Clock Skew Scheduling, Kluwer Academic Pub., 2000.

[11] R. G. Meyers and Ye Qiubo, "Incorporation of Zeland's IE3D in the microwave and RF classroom," Proc. of the Antennas and Propagation Society International Symp., vol. 1, pp. 688-691, June 2002.

[12] J. R. Phillips and M. Kamon, "An FFT-base Approach to Including Non-deal Ground Planes in a Fast 3-D Inductance Extraction Program," Proc. of the IEEE CICC, pp. 8.3.1-8.3.4, 1993 .

[13] A. E. Ruehli, "Inductance Calculations in a Complex Integrated Circuit Environment," IBM Joumal of Research and Development, pp 1750-1758, Sep. 1994.

[14] Semiconductor Industry Association, International Technology Roadmap for Semicondutors, 1999

[15] L. M. Silveira, M Kamon and J. K. White, "Efficient reduced-order modeling of frequency-dependent coupling inductances associated with 3-D interconnect structures, "Proc. of European Design and Test Conference, March 1995.

[16] Z. Zhu, B. Song, and J. K. White, "Algorithms in FastImp: A fast and wideband impedance extraction program fro complicated 3D geometries," IEEE Trans. ComputerAided Design, vol. 24, no. 7, pp. 981-998, July 2005. 